\title{
Aplicación del KAHOOT para la mejora de la adquisición de los objetivos del aprendizaje en el alumnado de Fisiología de los órganos del lenguaje y la audición
}

Jose Ignacio Priego Quesada ${ }^{\mathrm{ab}}$, Irene Jiménez Pérez ${ }^{\mathrm{ab}}$, Rosa $\mathrm{M}^{\mathrm{a}}$ Cibrián Ortiz de Anda $^{a}$, Rolando González-Peña ${ }^{a}$, Rosario Salvador Palmer ${ }^{a}$

${ }^{a}$ Grupo de Investigación en Física Médica (GIFIME), Departamento de Fisiología, Facultad de Medicina y Odontología, Universitat de València ${ }^{\text {}}$ Grupo de Investigación en Biomecánica aplicada al Deporte (GIBD), Departamento de Educación Física y Deportiva, Facultad de Ciencias de la Actividad Física y el Deporte, Universitat de València. Emails: j.priego.gibd@gmail.com; i.jimenez.gibd@gmail.com; rosa.m.cibrian@uv.es; Rolando.J.Gonzalez@uv.es; Rosario.Salvador@uv.es.

\begin{abstract}
One of the most used applications as a strategy of gamification in the classroom for the improvement of the learning process is KAHOOT. However, there are not many studies that have evaluated the repercussion of the application of sessions of this game at the end of the subject in the results of the final evaluation. In this way, the application of two complete sessions of KAHOOT was carried out at the end of the subject "Physiology of the Organs of Language and Hearing" of the Degree in Speech Pathology of the Universitat de València (course 2016-2017), with the objective of reviewing the contents related to the learning objectives of the subject. Two questionnaires, one for each session, were created consisting of 24 test questions with a duration of 1 and a half hour. The approval number of students of the subject was compared with the implementation of the KAHOOT session with respect to the two previous courses without innovation, observing an increase of $8 \%$. More specifically, the percentage of students with higher qualifications increased, while that of lower qualifications decreased. The analysis of this implementation in the qualifications of the present and future results will corroborate the results of this work.
\end{abstract}

Keywords: Gamification; web application; learning process; questionnaire; contents; motivation; evaluation results. 
Aplicación del KAHOOT para la mejora de la adquisición de los objetivos del aprendizaje en el alumnado de Fisiología de los órganos del lenguaje y la audición

\section{Resumen}

Una de las aplicaciones más utilizadas como estrategia de gamificación en el aula para la mejora del proceso de aprendizaje es el KAHOOT. Sin embargo, no existen muchos estudios que hayan evaluado la repercusión de la aplicación de sesiones de este juego al final de la asignatura en los resultados de la evaluación final. De esta forma, se llevó a cabo la aplicación de dos sesiones completas de KAHOOT al final de la asignatura “Fisiología de los órganos del lenguaje y la audición” del Grado de Logopedia de la Universitat de València (curso 2016-2017), con el objetivo de repasar los contenidos relacionados con los objetivos del aprendizaje de la asignatura. Se desarrolló en cada sesión un cuestionario compuesto de 24 preguntas tipo test con una duración de 1 hora y media. Se comparó el número de aprobados con la implementación de la sesión de KAHOOT respecto a los dos cursos anteriores sin innovación, observándose un aumento de un 8\%. De forma más específica, el porcentaje de alumnado con calificaciones altas aumentó, mientras que el de calificaciones bajas disminuyó. El análisis de dicha implementación en los resultados del presente y futuros cursos permitirá corroborar los resultados de este trabajo.

Palabras clave: gamificación, aplicación web, proceso de aprendizaje, cuestionario, contenidos, motivación, resultados de evaluación. 


\section{Introducción}

Para cualquier docente, uno de los objetivos principales de su proceso de enseñanza es conseguir que el máximo número de su alumnado alcance los objetivos de aprendizaje de la asignatura. Si el proceso de evaluación está correctamente diseñado, cuanto mayor sea el nivel alcanzado por el alumnado, se obtendrán mejores cualificaciones finales. En este sentido, diferentes estrategias se han explorado en las últimas décadas con el objetivo de mejorar el proceso de aprendizaje, siendo una de estas la gamificación.

La gamificación tiene como objetivo utilizar dinámicas del juego en contextos que no son propios del juego (empresas, aulas, etc.) (Deterding et al. 2011). Uno de los contextos en los que más se ha aplicado es en la educación, en la que se busca introducir elementos del juego en el diseño de los procesos de enseñanza (Bellotti et al. 2013). Se ha observado que la aplicación de la gamificación en las aulas propicia mejoras en los comportamientos cognitivos, afectivos y sociales dentro de la clase (Lee y Hammer 2011). Además, estos juegos suelen tener un componente de competición que aumenta el nivel de atención del alumnado (Bicen y Kocakoyun 2017). Entre los diferentes juegos de gamificación utilizados en las aulas, el KAHOOT es uno de los más empleados por la comunidad docente.

El KAHOOT es una aplicación web con la que el docente puede crear cuestionarios tipo test para que el alumnado conteste durante la clase utilizando sus teléfonos móviles, ordenadores o "tablets". Algunos aspectos positivos del KAHOOT es que es una aplicación gratuita, divertida, e intuitiva. El KAHOOT ha sido utilizado para reforzar contenidos impartidos en clases anteriores o para repasar al final de la clase los conceptos clave enseñados durante la sesión. Sin embargo, no existen muchos estudios que hayan evaluado la aplicación del KAHOOT al final de la asignatura, para repasar los contenidos relacionados con los objetivos del aprendizaje.

\section{Objetivos}

El objetivo del presente trabajo fue analizar los resultados en la evaluación final del alumnado de la asignatura "Fisiología de los órganos del lenguaje y la audición” del Grado de Logopedia de la Universitat de València, tras la aplicación de dos sesiones completas de KAHOOT al final de la asignatura, con el objetivo de repasar los contenidos relacionados con los objetivos del aprendizaje de la asignatura.

\section{Desarrollo de la innovación}

\subsection{Alumnado sobre el que se ha desarrollado la innovación}

La innovación se llevó a cabo en el curso 2016-2017 en la asignatura "Fisiología de los órganos del lenguaje y la audición” del Grado de Logopedia, de la Universitat de València. Dicha asignatura se imparte en el primer curso del grado. Todo el alumnado del curso, es decir, 80 estudiantes matriculados en la asignatura, participaron en la innovación. 


\subsection{Diseño de la innovación}

La innovación se llevó a cabo al final de la impartición teórica, siendo las dos últimas prácticas de la asignatura. Se desarrollaron dos sesiones de 1 hora y media de KAHOOT. Una de las sesiones estuvo dedicada a las bases de la audición y la fonación y la otra a las bases de la electrofisiología, cubriendo así el temario de la asignatura. Se utilizó la subdivisión en grupos de prácticas (unos 20 estudiantes en el aula). El cuestionario para ambas sesiones estuvo compuesto de 24 preguntas tipo test con entre 2 y 4 posibles respuestas (Fig. 1), cubriendo entre las dos sesiones el temario de la asignatura. Antes de la sesión se insistió al alumnado que era de gran importancia que estudiasen el temario y apuntes de la asignatura de cara a cada una de las sesiones. Las preguntas fueron confeccionadas conjuntamente por el profesorado de la asignatura teniendo en cuenta los siguientes aspectos:

a) Debían tratar contenidos y conceptos clave que tuviesen que ver con los objetivos de aprendizaje desarrollados en la guía docente de la asignatura.

b) Cada pregunta fue confeccionada para poderse responder con claridad en 30 segundos, que fue el tiempo máximo estipulado en la aplicación.

c) En ningún caso debían ser preguntas semejantes a las que aparecerían en el examen final de la asignatura.

Durante la sesión, se llevó a cabo el siguiente orden de acciones:

a) Dividir a la clase en 5 grupos de 4 estudiantes cada uno. La elección de 5 grupos vino motivada por la componente de juego que tiene esta modalidad de enseñanza. Como se indicará posteriormente en "visualización de los resultados", a lo largo del cuestionario va apareciendo un ranking de mejores resultados pero sólo se visualizan 5 grupos. Por lo tanto, para que los estudiantes vean su evolución no interesa más de estos 5 grupos.

b) Se utiliza para cada grupo un pseudónimo y se trabaja en grupo con lo que ningún estudiante queda explícitamente señalado si no resulta ganador.

c) Para cada una de las preguntas, el protocolo fue:

- Tiempo para mostrar la pregunta y que el alumnado la discutiera y respondiera.

- Visualización de la respuesta correcta y las respuestas erróneas. En este tiempo, el/la profesor/a debía aprovechar para aclarar la respuesta correcta. Si el/la profesor/a detectaba un déficit por parte de los estudiantes en algún concepto o contenido, se procedía a una explicación más detallada.

- Visualización de los resultados. La aplicación permite mostrar en cada pregunta el ranking de los estudiantes teniendo en cuenta haber acertado la pregunta, así como el tiempo empleado en contestar. Tras este ranking por preguntas, también se mostraba el ranking del cómputo total de preguntas contestadas hasta el momento. En este tiempo, se fomentaba la competición para aumentar la motivación del alumnado. También se aprovechaba para utilizar el humor como herramienta de motivación, y para que los grupos que

(c) EY-NC-ND 2018, Universitat Politècnica de València

Congreso IN-RED (2018) 
fallasen no se sintiesen avergonzados y entendiesen que el objetivo de la sesión era mejorar su proceso de aprendizaje.
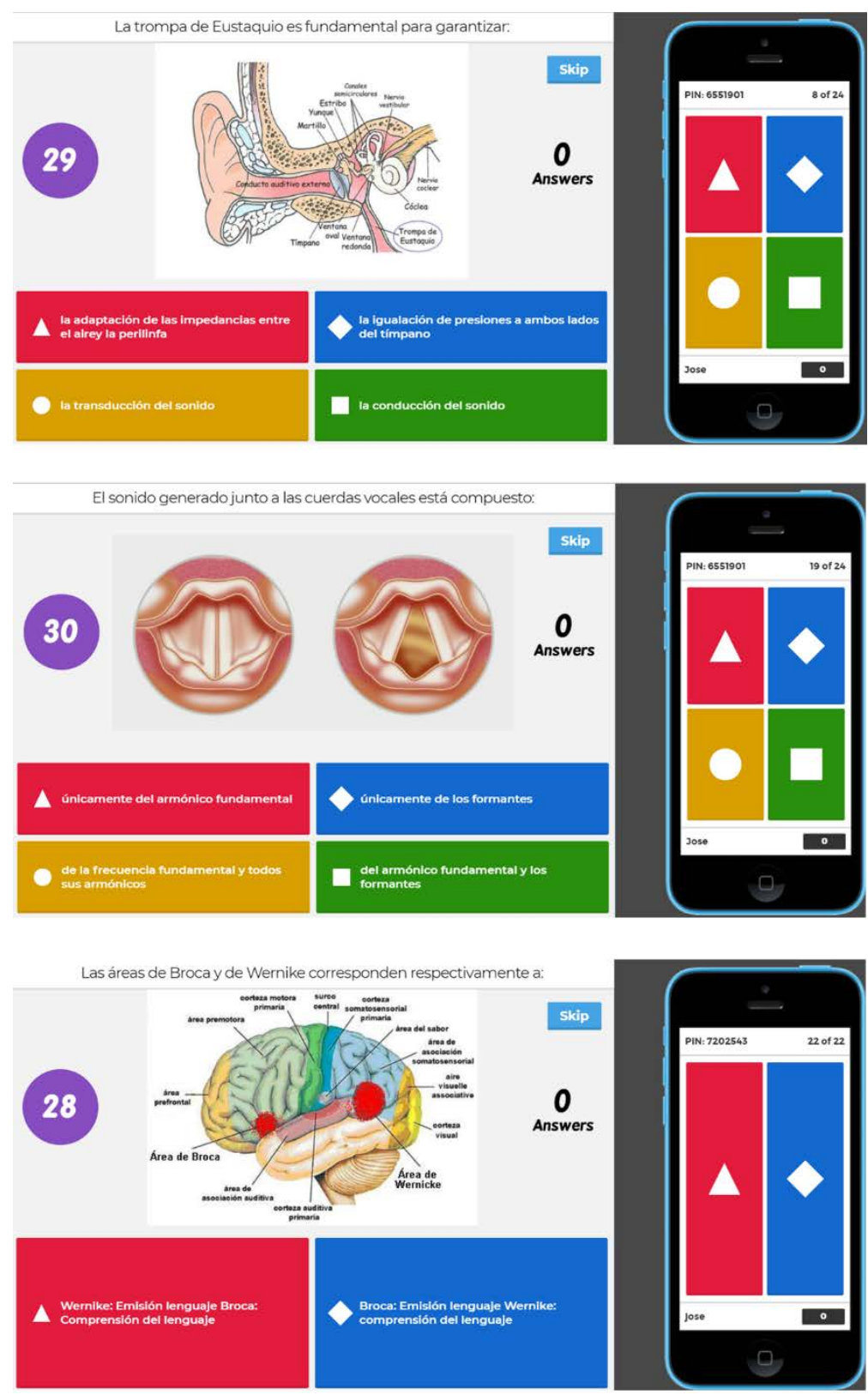

Fig. 1 Ejemplos del cuestionario KAHOOT empleado.

\subsection{Análisis de los datos}

Con el objetivo de analizar la eficacia de la innovación implementada, se compararon los resultados de la evaluación final de los estudiantes del curso 2016-2017, con los resultados 
de los dos cursos anteriores en los que no se realizaba la sesión KAHOOT. En los dos cursos previos, la asignatura tuvo 74 estudiantes matriculados en cada curso, y en el curso 2016-2017 el número de estudiantes matriculados fue de 80. Para dicho análisis, se tuvo en cuenta tanto el porcentaje de aprobados total, el porcentaje de presentados en la primera convocatoria, así como el porcentaje para cada una de las calificaciones: suspenso, aprobado, notable, sobresaliente/matrícula. La dificultad del examen de la evaluación final fue similar en los tres cursos evaluados.

\section{Resultados}

La implementación de las sesiones de KAHOOT al final de la asignatura, con el objetivo de repasar los contenidos relacionados con los objetivos del aprendizaje, aumentó en aproximadamente un $8 \%$ los estudiantes aprobados respecto a los dos cursos anteriores (Fig. 2).

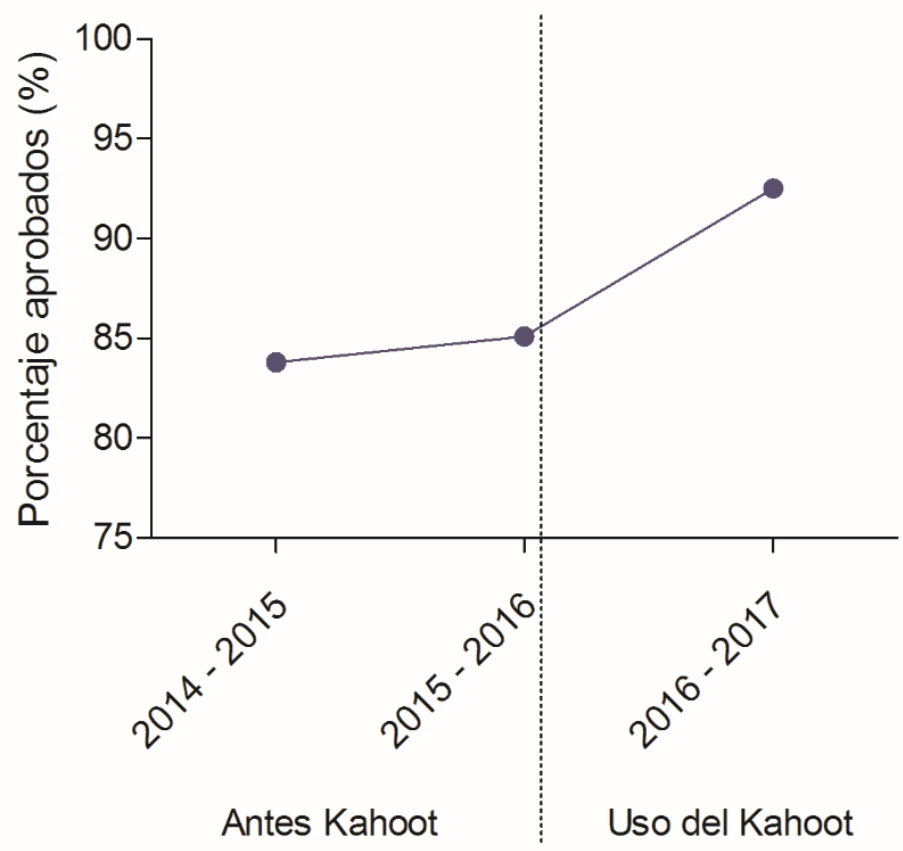

Fig. 2 Porcentaje de aprobados en los dos cursos anteriores a la implementación de la sesión de KAHOOT y en el curso en el que fue implementado.

De manera más específica, se puede observar como la implementación de las sesiones de KAHOOT cambió la distribución de los estudiantes en las diferentes calificaciones (Fig. 3). El porcentaje de alumnado que obtuvo calificaciones altas aumentó (notables y sobresalientes/matrículas) y el porcentaje que obtuvo calificaciones bajas disminuyó (suspensos y aprobados). De esta forma, el porcentaje de estudiantes que obtuvieron notable aumentó con la implementación de la sesión de KAHOOT en aproximadamente un

(cc) EY-NC-ND 2018, Universitat Politècnica de València

Congreso IN-RED (2018) 
20\%, y los que obtuvieron sobresaliente/matrícula en un $8 \%$. Mientras que el porcentaje de estudiantes que suspendían la asignatura se redujo en aproximadamente un $8 \%$, y los que obtenían aprobado se redujo en un $20 \%$ en comparación con los dos cursos anteriores. Estos resultados además son muy positivos si se tiene en cuenta el porcentaje de estudiantes presentados en la primera convocatoria. El porcentaje de estudiantes presentados en la primera convocatoria fue del 81\% en el curso 2014-2015, del 85\% en el curso 2015-2016, y del 89\% en el curso 2016-2017. Por lo tanto, la mejora del porcentaje de calificaciones altas estuvo acompañada de un aumento del porcentaje de estudiantes presentados en primera convocatoria.

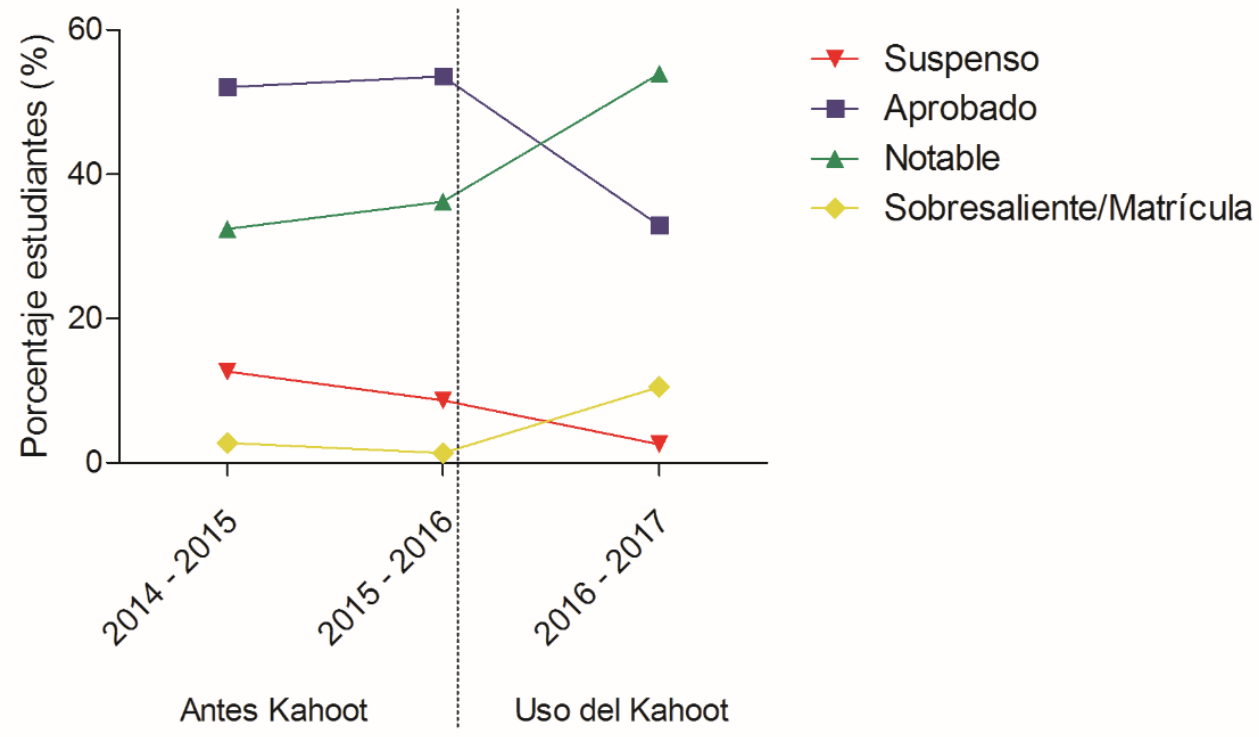

Fig. 3 Porcentaje de estudiantes que obtuvieron las diferentes calificaciones específicas en los dos cursos anteriores a la implementación de la sesión de KAHOOT y en el curso en el que fue implementado.

\section{Conclusiones}

La utilización de KAHOOT al final de la asignatura "Fisiología de los órganos del lenguaje y la audición” con el objetivo de aclarar, repasar y fijar los contenidos relacionados con los objetivos del aprendizaje resultó beneficiosa aumentando, tanto el porcentaje de estudiantes aprobados en un $8 \%$, como los porcentajes correspondientes a las calificaciones más altas (notable en un $20 \%$ y sobresaliente/matrícula en un $8 \%$ ).

El análisis de dicha implementación en los resultados del presente y futuros cursos permitirá corroborar los resultados de este trabajo.

\section{Referencias}

BELlOtTI, F., BERTA, R., DE GLORIA, A., LAVAGNino, E., ANTONACI, A., DAGNiNO, F.M., y OTT, M. (2013). “A gamified short course for promoting entrepreneurship among ICT 
Aplicación del KAHOOT para la mejora de la adquisición de los objetivos del aprendizaje en el alumnado de Fisiología de los órganos del lenguaje y la audición

engineering students”. En: 13 th International Conference on Advanced Learning Technologies (ICALT). IEEE, 31-32.

BICEN, H. y KOCAKOYUN, S. (2017). "Determination of University Students’ Most Preferred Mobile Application for Gamification” en World Journal on Educational Technology: Current Issues, 9, 1, 18-23.

DETERDING, S., DIXON, D., KHALED, R., y NACKE, L. (2011). "From game design elements to gamefulness: defining gamification”. En: Proceedings of the 15th international academic MindTrek conference: Envisioning future media environments. ACM, 9-15.

LEE, J.J. y HAMMER, J. (2011). “Gamification in education: What, how, why bother?” en Academic exchange quarterly, 15, 2, 146.

(cc) EY-NC-ND 2018, Universitat Politècnica de València 